Int. J. Dev. Biol. 59: 63-71 (2015)

doi: $10.1387 / \mathrm{ijdb} .150070 \mathrm{ev}$

\title{
Development and programed cell death in the mammalian eye
}

\author{
ELENA VECINO* and ARANTXA ACERA \\ Experimental Ophthalmo-Biology Group (GOBE), Department of Cell Biology and Histology, \\ University of the Basque Country (UPV/EHU), Leioa, Vizcaya, Spain
}

\begin{abstract}
Programmed cell death (PCD) is a major mechanism for patterning of a variety of complex structures. Cells are initially organized into fairly loose patterns; then, selective death removes the cells between pattern elements to create the correct structures, as a sculptor removes some material to reveal the hidden image. The life or death of a cell is mostly affected by extracellular signals because the intracellular machinery responsible for PCD is constitutively expressed in most animal cells. The optic vesicle originates during gastrulation when the endoderm and mesoderm interact with the adjacent prospective head ectoderm to create a lens. To be formed correctly, the lens must have a precise spatial relationship with the retina. Ganglion cells are the first neurons to be differentiated in the retina. Vertical networks in the inner and outer retina are later interconnected when bipolar cells are formed and connections with ganglion cells are established.This sequential pattern of retinal circuit development is common across vertebrate species. During development of the retina, far more neurons are generated than are ultimately needed with almost one half of them undergoing PCD shortly before establishing meaningful contacts within their targets. However, apoptosis in other eye tissues is not a key event but rather a refinement. Thus, for the final development of the cornea, the control of keratocyte proliferation is more important than cell death events. The molecular mechanisms underlying apoptotic cell death have been conserved throughout evolution; however further investigations are needed to understand the key mechanisms of PCD in different tissues during development.
\end{abstract}

KEY WORDS: retina, cornea, lens, ciliary body, iris, programed cell death, eye, development

"By the time I was born, more of me had died than survived."

Lewis Thomas, 1992

The emergence of new structures in tissues is made possible by selective cell death during development. By imposing patterned cell death across a tissue, boundaries can be created and tightened. Programmed cell death (PCD) is a major mechanism for patterning of a variety of complex structures. Cells are initially organized into fairly loose patterns; then, selective death removes the cells between pattern elements to create the correct structures, as a sculptor removes some material to reveal the hidden image. The order to remove certain cells comes as signals at the cell surface, which are responsible for tissue patterning and induce the cell death machinery in an organized way.

One can wonder why should the nature, having selected the most economical way of evolutionary survival, create cells only to remove them later. One of the most puzzling phenomena in the development of the nervous system is neuronal cell death. In many parts of the vertebrate central and peripheral nervous systems, over half of the neurons die during the normal course of development. Moreover, there are large differences between species. For instance, in the cat retina, around 80 percent of the retinal ganglion cells die, while in the chicken retina, only 40 percent. In the retinas of fishes and amphibians, no retinal ganglion cells appear to die (Patterson, 1992).

An initial over proliferation of cells followed by the elimination of the surplus is a common strategy when complex patterns, such as neural connectivity, must be imposed on populations of cells whose numbers cannot be precisely specified by the genome. In Drosophila, a geometrically regular hexagonal array of unit eyes,

Abbreviations used in this paper: PCD, programmed cell death.

\footnotetext{
Accepted: 6 May 2015.

ISSN: Online 1696-3547, Print 0214-6282

*Address correspondence to: Elena Vecino. Experimental Ophthalmo-Biology Group (GOBE), Department of Cell Biology and Histology University of the Basque Country (UPV/EHU), Leioa E-48940, Vizcaya, Spain. Tel. 34+946-012-820. E-mail: elena.vecino@ehu.eus - web: www.ehu.eus/GOBE 
or ommatidia, is created as approximately 15 million surplus cells in the retinal epithelium are eliminated by cell death. Near the end of the first third of pupal life, the fruit fly retina has an exact cellular order; each of the eight photoreceptors and a dozen accessory cells that make up an ommatidium occupy a precise location in an extended cellular lattice. This makes the eye a sensitive detector of mutations that prevent normal cell death.

The life or death of a cell is mostly affected by extracellular signals because the intracellular machinery responsible for programmed cell death is constitutively expressed in most animal cells. In vertebrates, the timing and location of apoptosis in the lens vesicle and the optic stalk suggest their involvement in the separation of the lens vesicle from the surface ectoderm and narrowing of the proximal optic vesicle to form the stalk.

To understand the anatomy and function of the eye, it is necessary to follow its embryonic development. The eyes of all vertebrates develop in a pattern that produces an "inverted" retina, in which the initial detection of light rays takes place in the outermost region. Early eye development is induced by signals from different tissues involved in its formation. The neural tube (neuroectoderm) forms the retina, iris, and optic nerve. The surface ectoderm forms the lens and corneal epithelium. The mesenchyme has two sources; one of them is the mesoderm, giving rise to the fibrous, vascular, and muscle elements of the eyeball. The other source is the ectomesenchyme derived from neural crest cells, which forms the vitreous body, sclera, and corneal endothelium. Once the formation of the
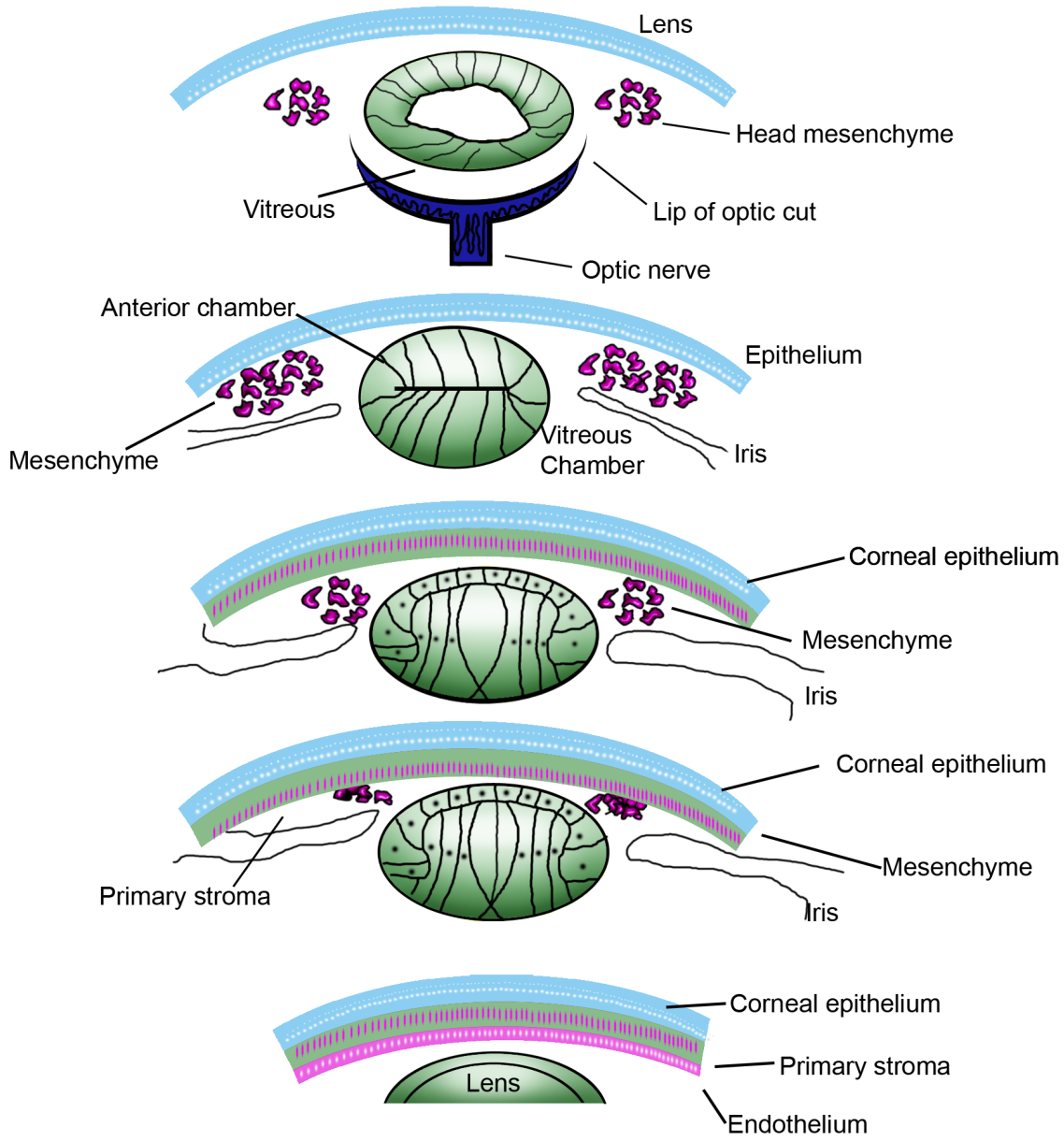

lens has started, the expanding optic vesicle begins to invaginate to form a cup-shaped structure and folds along its central line, thus enclosing a small portion of angiogenic mesenchyme. This part of mesenchyme forms the hyaloid artery and vein, which supply the forming lens and later, at the fully formed stage, will become the central artery and vein of the retina.

The eye establishes an anatomical connection with the diencephalon, the part of the embryonic neural tube apparent from the beginning of the development. During tissue morphogenesis, the coordination of cell migration, proliferation, and cell death are under tight spatiotemporal molecular control requiring cooperation of distinct signaling pathways. Genetic screens have identified molecularly diverse genes that mediate localized epithelial fusion processes in the developing neural tube, palate, and eye. However, how these genes exert their influence at the cellular level is still poorly understood (Yu, H., et al., 2010).

The optic vesicle originates during gastrulation when the involuting endoderm and mesoderm interact with the adjacent prospective head ectoderm to create a lens. To be formed correctly, the lens must have a precise spatial relationship with the retina. As the optic vesicle becomes the optic cup, two layers differentiate in different directions. The cells of the outer layer produce melanin (one of the few tissues other than the neural crest cells that can form this pigment) and ultimately become the pigmented retina. The cells of the inner layer proliferate rapidly and generate a variety of glia, ganglion cells, interneurons, and light-sensitive photoreceptor neurons. Collectively, these cells constitute the neural retina. The retinal ganglion cells are the neurons whose axons send electrical impulses to the brain. Their axons meet at the base of the eye and travel down the optic stalk. This stalk then transforms into the optic nerve (Fig. 1).

A group of transcription factors, Six3, Pax6, and $\mathrm{R} x 1$ are expressed in the anterior tip of the neural plate. This single domain will later split into the bilateral regions that will form the optic vesicles. Again, we see similarities between the Drosophila and the vertebrate nervous system, for these three proteins are also necessary for the formation of the Drosophila eye. The Pax6 protein is especially important in the development of the lens and retina. Indeed, this protein is a common denominator for photoreceptive cells in all phyla. If the mouse Pax6 gene is inserted into the Drosophila genome and activated randomly, Drosophila eyes form in the cells expressing the mouse gene.

Fig. 1. Lens and corneal development. The optic cup induces formation of the lens. The growth factors from the anterior and vitreous chambers cause dorsal lens cells to differentiate and ventral lens to proliferate. Then lens induces overlying ectoderm to columnar and secretory epithelium. Mesenchymal cell secretions cause the stroma to shrink. Under the inductive influence of the lens, the corneal epithelium differentiates and secrets a primary stroma consisting of collagen layers. Endothelial cells secrete hyaluronic acid causing the stroma to swell, and mesenchymal cells enter. The stroma then eventually becomes the cornea. 
While Pax6 is also expressed in the murine forebrain, hindbrain, and nasal placodes, the eyes seem to be most sensitive to its absence. In humans and mice, Pax6 heterozygotes have small eyes, while homozygotic mice and humans (and Drosophila) lack eyes altogether (Quiring et al., 1994).

\section{Molecular pathways involved in eye development}

The best-known genes involved in morphogenesis of the human eyeball are Pax 6 and Pax 2 genes located on chromosome 11 and 10, respectively. The Pax6 gene has been called "the master gene for eye morphogenesis control" because it triggers a chain of actions involving approximately 2,500 genes that guide the development of this organ (Dahl et al., 1997). However, more and more genes and molecular mechanisms involved in the development of various ocular tissues are being discovered. In different stages of development, there are some "critical steps" such as the formation of the eye field, specification of the neural retina and retinal pigment epithelium (RPE), proliferation, neurogenesis, lamination and development of retinal connectivity, differentiation of the lens, ciliary body and iris, and vasculogenesis. A disruption of any of these steps can result in severe eye defects and ultimately result in blindness. Thus, the development of ocular tissues must be precisely coordinated and is controlled by intrinsic and extracellular factors (e.g., hedgehog, fibroblast growth factor (FGF), TGF $\beta$ ) (Degterev A, Yuan J, 2008).

Wnt/Frizzled pathway has a very important role in the development of the different structures of the eye. This pathway is involved in many processes such as polarity, cellular proliferation and differentiation, axonal outgrowth, and cellular maintenance, especially in the central nervous system (CNS) (Ang SJ et al., 2004; Liu H, 2006).

Gain- and loss-of-function studies reveal that Wnt/Frizzled pathways are involved in coordinating critical processes during ocular tissue development. Ocular development starts with the formation of an area of cells in which are expressed eye precursors in the anterior neural plate during late gastrulation. These cells expresses transcription factors (EFTFs; e.g., $R x$, Pax6, Six3, Optx2) that establish a genetic network to control the eye specification, which requires concomitant inhibition of BMP, Nodal, and Wnt/ $\beta$-catenin signaling in the anterior neural plate (Yamamoto A, et al., 2005; Zuber ME, et al., 2003).

Several lines of evidence indicate that non-canonical Wnt signaling is essential for formation and/or maintenance of the eye field, by mediating morphogenetic movements. Wnt antagonists are expressed in the neural plate to permit the development of the forebrain, including the eye field, by suppressing Wnt/ $\beta$-catenin signaling. Very close to the caudal border of the eye field, Wnt11 and Wnt4 are expressed and act via $F z d 5$ and $F z 3$ to activate non-canonical Wnt pathways in the eye field (Cavodeassi F, et al., 2005). This mechanism permits morphogenetic movements of retinal precursors into the eye field and promotes the expression of eye-field-specific transcription factors, e.g., Pax6 and $R x$.

\section{The retina}

The inner layer of the optic cup thickens and initiates differentiation into neurons and photoreceptor cells of the neural retina. The outer layer of the optic cup is thin and eventually becomes the pigment layer of the retina in which the Otx-2 gene is expressed. In the formation of the optic cup, the distinction between the neural retina and RPE depends on molecular events during the early stages eye formation. Under the influence of Pax6 gene, the distal portion of the optic vesicle invaginates to form the inner wall of the optic cup. The neural retina also expresses the transcription factor CHX-10 (De Melo et al., 2003). The structure consists of multiple layers formed by three types of neurons: photoreceptors, bipolar and ganglion cells. The ganglion cells emit extensions that exit the eye to form the optic nerve and reach the brain.

Synaptic connections of the adult retina are organized into distinct laminae. In the outer retina, photoreceptors contact horizontal cells and bipolar cells within a single lamina, the outer plexiform layer (OPL), see Fig. 2. Within the inner retina, synapses between retinal ganglion cells and their presynaptic partners, the amacrine and bipolar interneurons, are localized in the inner plexiform layer (IPL).

Ultrastructural studies (Fisher, 1979) have suggested that synaptogenesis between the major neuronal classes of the vertebrate retina occurs in four major phases. Retinal ganglion cells are the first class to differentiate, and then form the earliest functional circuits in the IPL of the developing retina. Shortly afterwards, horizontal cells and photoreceptors differentiate and contact each other in the outer retina, forming the OPL. The vertical networks in the inner and outer retina are later interconnected when bipolar cells are formed and connections with ganglion cells are established. This sequential pattern of retinal circuit development is common across vertebrate species. However, the temporal separation between inner and outer retinal circuit development varies, ranging from a few hours in animals such as the zebrafish to many days or weeks in mammals (Fig. 2).

However, not all progenitor cells or newly post-mitotic neurons in the local micro-environment may be competent to respond to the
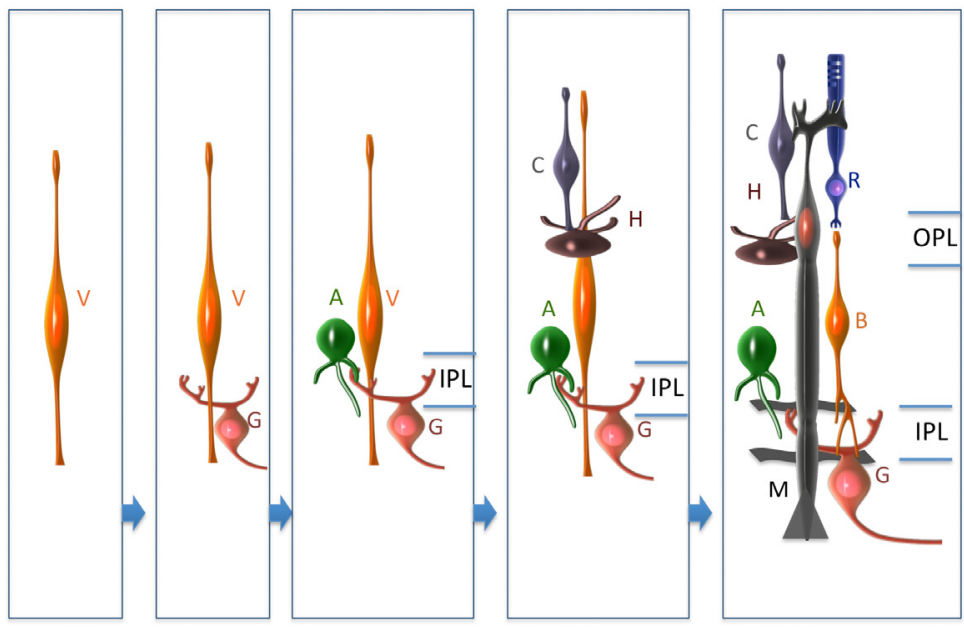

Fig. 2. Phases of retina cell differentiation. Summary of the times of appearance of differentiated cells in the fetal retina. Prior on the onset of neurogenesis the retina is a pseudostratified columnar epithelium with ventricular cells (V). Soon after their final mitosis, the firs cells migrate to their appropriate position in the proximal retina. The differentiation of the cells follows an order first ganglion cells (G), then amacrine cells (A), horizontal cells (H), cones (C), bipolar cells (B) and rods (R) and finally Müller glia (M). As the cells differentiate, the plexiform layers are formed, first the inner plexiform layer (IPL) and then the outer plexiform layer (OPL). 
signals. The field of developmental neuroscience has undergone explosive growth in the past two decades as a result of the recent advances in molecular biology. These advances have yielded fresh insights into the control of neuronal fate and differentiation and regulatory mechanisms triggered upon proliferation. A variety of transcription factors, mostly of the basic helix-loop-helix (bHLH) and homeodomain families, are expressed by subsets of retinal progenitor cells or their post-mitotic progeny (the retinal precursor cells) at particular periods during the retinal development (Ohsawa \& Kageyama, 2008). Indeed, a simple inactivation or misexpression of many of these genes can lead to striking alterations in the cellular composition of the retina. In some cell-type specific cases, these changes lead to an alternative fate or a failure to differentiate correctly and are often followed by cell death.

During retinal development, two phases of programed cell death are commonly observed. Neuronal cell death in the late phase of apoptosis is associated with a lack of trophic factors. In contrast, survival of neural cells and developing neurons during the early phase of apoptosis is regulated by neurotrophic factors and/or by interactions of their afferents with neighboring glial cells, so that the number of afferent neurons matches available sites in the targetspace (Clarke, 1985; Oppenheim, 1991; Linden, 1994). Electrical activity can also influence the early survival of both neurons and glial cells by activating the appropriate intrinsic mechanisms (Meyer-Franke et al., 1998). The evidence for the dependence of apoptosis on cell-cell interactions has been obtained by experimental manipulation of the developing nervous system. Moreover, the characterization of various factors derived from neurons or glia has revealed some of the cues essential for the PCD. Pharmacological manipulation and generation of transgenic animals have helped to elucidate, at least in part, the mechanisms and timing of the apoptotic processes in the developing retina.

The precise magnitude and timing of retinal cell death has not been completely characterized. To elucidate these processes, we have to analyze the retinal cell death in a quantitative and standardized manner. However, it is very difficult to estimate the

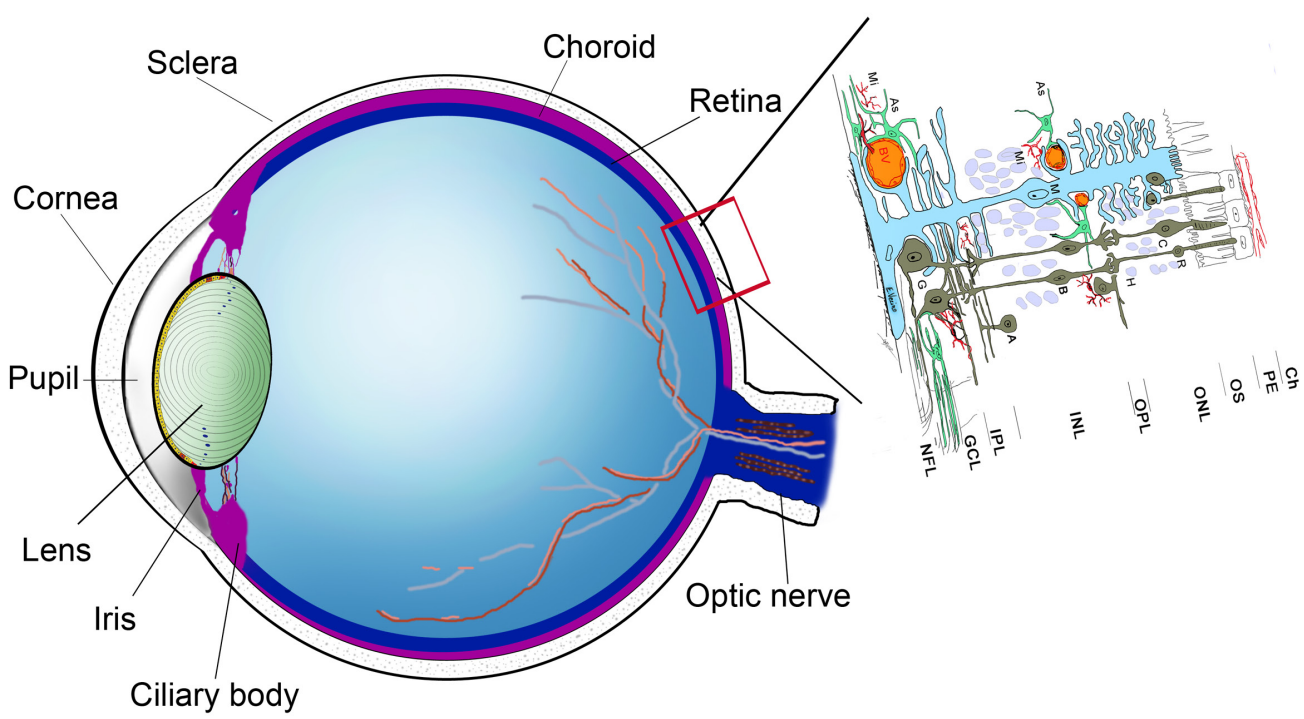

Fig. 3. Different parts of the human eye. Abbreviations: Ch, choroid; GCL, ganglion cell layer; INL, inner nuclear layer; IPL, inner plexiform layer; NFL, nerve fiber layer; ONL, outer nuclear layer; OPL, outer plexiform layer; OS, outer segments of the photoreceptors; PE, pigment epithelium. magnitude of cell death in the retina since the cells cannot be specifically labeled before they commit themselves to their definitive phenotype. For this reason, most of the quantitative data for cell death in the developing retina have come from the studies of retinal ganglion cells (Bähr, 2000; Isenmann et al., 2003). In the rat retina, up to $90 \%$ of recently born retinal ganglion cells (RGCs) die during retinal development (Galli-Resta and Ensini, 1996). Interestingly, the actual fraction of apoptotic cell profiles is less than $1 \%$, according to the results obtained by Perry et al., using instantaneous detection methods (Perry et al., 1983). The percentages and patterns of cell death in the retina vary between the species (Vecino et al., 2011). It has been postulated that cell death is caused by the competition for adequate arborization space. If the number of retinal afferent fibers is higher than the number of available tectal termination sites, supernumerary fibers might degenerate (Rager and Rager, 1978).

Avariety of proapoptotic stimuli, including extracellular and intracellular signals, can lead to apoptotic cell death. The extracellular signals include tumor necrosis factor (TNF), Fas ligands (FasL), and the absence of trophic factors. DNA damage and endoplasmic reticulum (ER) stress are examples of intracellular proapoptotic signals. These specific signals activate the intracellular pathways, resulting in biochemical and morphological changes in the cell. Caspases belong to the family of proteases with a cysteine at their active site and cleave their target proteins at specific aspartic acid residues. These enzymes are typically activated during the early stages of apoptosis (Tornberry and Lazebnik, 1998). Caspases are synthesized in the cell as inactive precursors, procaspases, which are, usually, activated via proteolytic cleavage by another member of the caspase family, forming an amplifying proteolytic cascade. These proteases cleave key cellular substrates required for normal cellular functions, including structural proteins in the cytoskeleton and nuclear proteins such as DNA repair enzymes. Caspases can also activate other degradative enzymes such as DNases, which cleave DNA in the nucleus. The activation of these powerful death effectors is a critical process. Mitochondria play an important role in the regulation of cell death by releasing cytochrome C. Cytochrome C, Apaf-1, and ATP form a complex with procaspase-9, leading to activation of this caspase and triggering the caspase cascade.

The relationship between apoptotic and proliferating cells has been demonstrated in the developing cerebral cortex of the rat (Thomaidou et al., 1997). Thus, the same substance can exert two opposite actions to create one structure, as it has been shown in the chicken retina (Trousse F et al., 2001). The bone morphogenetic proteins (BMP) are a large subclass of the transforming growth factorB superfamily of soluble proteins. These proteins are expressed throughout embryogenesis and play an important role in the neural plate induction or dorso-ventral patterning of neural 
tube; they are also involved in the proliferation and differentiation of many organs. BMP4 also has other roles during the eye morphogenesis. In the chicken, BMP4 expressed in the dorsal prospective neural retina is responsible for the apoptotic cell death with a characteristic spatiotemporally restricted pattern. Inhibition of BMP4 activity leads to a further clear reduction in the eye size; this is associated with a decrease in the number of cells undergoing mitosis in the dorsal optic cup. Thus, BMP4 signaling controls the size of the dorsal optic cup by regulating cell proliferation and apoptosis. Spatiotemporally restricted patterns of apoptosis in the chickens and mice are similar but not identical. However, the programed cell death in the dorsal optic cup is more pronounced in the chicken. As BMP4 increases the rate of cell proliferation with a consequent time-dependent programed cell death, it might control the asymmetric growth of the chick optic cup, which has an enlarged dorsal portion. When this asymmetry is established, the restricted (dorsal) activity is no longer required, and the molecules of the BMP4 signal transduction pathway (Smad1) begin to migrate to the remaining retinal neuroepithelium. The low level of apoptosis in the mouse dorsal optic cup, where the asymmetric dorsal portion of the eye is less pronounced than in the chicken, further supports this idea (Trousse F et al., 2001). These events and the activation and inhibition of certain genes sculpt the structure of the organs, including the shape of the retina.

\section{The ciliary body and the iris}

The peripheral rim of the optic cup forms the ciliary margin, which differentiates into the ciliary body and the iris. The ciliary body and the peripheral iris comprise the non-pigmented and pigmented ciliary epithelium that extends from the retina and RPE, respectively. It has been suggested that the ciliary body is specified at the optic vesicle stage in a transition zone between RPE and retina; the process is regulated by a tightly coordinated gradient of FGF and TGF-beta family factors (Dias da Silva MR, et al., 2007). Furthermore, the retinal stem cells have been identified in the ciliary body and the iris (Ahmad I, et al., 2004; Reh TA and Fischer AJ, 2006).

\section{The lens}

The formation of the lens placode, a thickened portion of the ectoderm, requires interactions between the distal optic vesicle and the overlying lens ectoderm (Hyer, 2003). During optic cup formation, the lens placode invaginates and closes, resulting in the separation of the lens vesicle. At this stage, the signals from the developing neural retina induce the elongation of cells closest to the posterior end of the lens vesicle towards the anterior end of the vesicle. These signals also induce the synthesis of crystallins. The elongating cells eventually fill in the lumen of the vesicle to form the primary fibers, which become the embryonic nucleus in the mature lens. The cells of the anterior portion of the lens vesicle give rise to the lens epithelium.

Additional secondary fibers are derived from epithelial cells close to the equatorial region of the lens. These cells lengthen to encircle the primary fibers. The new fibers grow longer than those in the primary layer, but as the lens becomes larger, the ends of the newer fibers cannot reach the posterior or anterior poles of the lens. The lens fibers that do not reach the poles form tight, interdigitating seams with neighboring fibers. These seams, called sutures, are readily visible. The suture patterns become more complex as more layers of lens fibers are added to the outer portion of the lens (Hyer, 2003). The lens continues to grow after birth and new secondary fibers are added as outer layers. The new fibers are generated from the equatorial cells of the lens epithelium, in a region referred to as the germinative zone. The lens epithelial cells elongate, lose contact with the capsule and epithelium, synthesize crystallin, and then finally lose their nuclei (enucleate) as they mature. During the development and through early adulthood, the addition of secondary lens fibers continues and the lens becomes increasingly ellipsoidal (Fig. 4). However, after the age of 20 , the lens grows gradually rounder; the iris is very important in this process since it limits the growth.

While many of the tissue interactions involved in lens induction have been defined, not much is known about the regulatory factors that mediate these events. Most progress has been made in the area of identification of key transcription factors that are expressed in the presumptive lens ectoderm and required for lens formation. Various genetic manipulations in Drosophila and mice, as well as studies of human mutations, have resulted in the identification of many different classes of transcription factors important in the lens development (Goudreau et al., 2004). The foremost among these is the highly conserved Pax6 gene, which appears to be at the top of the regulatory hierarchy. However, it is unclear how these key genes are regulated. Mutant mouse studies indicate that FGF (Faber et al., 2001) and bone morphogenetic protein 7 (BMP7) receptor signaling are required for lens induction and cooperate to promote Pax6 expression (Lang and McAvoy, 2004). The lens is an important model for investigation of cellular differentiation, partly because it allows the visualization of all developmental stages within a single sagittal section of the eye. The lens consists entirely of cells developing from the surface ectoderm-derived lens placode. These cells are divided into epithelial cells, forming a single layer of cuboidal epithelium immediately under the anterior lens capsule, and fiber cells, which make up the remainder of the lens. The primary fiber cells differentiate from the posterior hemisphere cells of the lens vesicle, and secondary fiber cells are continuously generated from epithelial cells approaching the lens equator. Lens fiber-cell differentiation follows a sequence beginning in the equatorial epithelial cells and continuing from periphery to the center, where the oldest fiber cells are found. The vitreous humor, which surrounds the posterior hemisphere of the lens, exhibits a potent fiber-cell differentiation activity (Beebe et al., 1980).

To date, most of the research has focused on the identification of the key molecules and specific signaling pathways that affect lens cell behavior. The impaired function of these molecules, and the dysregulation of their respective signaling pathways can cause many diseases. As FGFs play such a key role in lens biology, a 'gradient' of FGF stimulation in the eye may be critical for the regulation of distinct spatial lens cell processes. However, the molecules that inhibit FGF signaling may also be involved in these processes, in addition to their role in the regulation of anteroposterior levels of FGF bioactivity (Fig. 4). FGF signaling is important for lens formation. However, the stages at which FGFs mediate lens formation, the FGF types involved, the cells that secrete them, and the mechanisms of promotion of lens formation are not known. We found that the transcripts encoding several FGF ligands and the four classical FGF receptors are detectable in the lens-forming ectoderm at the 
time of lens induction. Conditional deletion of Fgfr1 and Fgfr2 from this tissue resultes in the formation of small lens rudiments that soon degenerate. Lens placodes lacking Fgfr1 and Fgfr2 were thinner than in wild-type embryos. Deletion of Fgfr2 increase cell death at the initiation of placode formation and concurrent deletion of Fgfr1 enhance this phenotype. These results suggest that the primary role of autocrine or paracrine FGF signaling is to provide the essential survival signals to the lens placode cells. At the onset of placode formation in Fgfr1/2 placode cells, the apoptosis has already increased; thus, there are no FGF signaling during the lens induction by the optic vesicle. Since the expression of proteins required for lens formation were not altered in these cells, it was concluded that FGF signaling from the optic vesicle is not required for the lens induction (García CM et al., 2011). Very little is known about the cell death in the lens during the development or the lens-size determination in different animals. However, the active caspase-3 has been found in normal lens epithelium and its level of expression is higher in the anterior pole of the lens. The probability of radial spatial distribution of labeling has been fitted with a logistic model. The gradual decrease of active caspase-3 labeling from the anterior pole to the periphery suggests that caspase-3 might be involved in the normal protein turnover (e.g., caused by incident light) (Talebizadeh $\mathrm{N}$ et al., 2014).

Apoptosis might have an important function during lens vesicle development. This unique organ is derived from the embryonic ectoderm via a complicated differentiation process in which apoptosis is involved. First, when the committed ectoderm thickens and invaginates, the number of cells required to form the lens vesicle is partially determined by apoptosis. Secondly, the separation of the lens vesicle from the corneal ectoderm is executed through apoptosis of the lens stalk cells. Finally, differentiation of the lens epithelial cells is controlled by the regulators that are mostly involved in control of apoptosis at multiple signaling steps. In the adulthood, the lens also grows and differentiates continuously. During the different stages of growth and differentiation in the adult lens, various stresses can induce apoptosis of the epithelial cells, leading to eventual non-congenital cataractogenesis (Zhang et al., 2010). Under normal physiological conditions, most of these cells have a relatively long life span. Alterations or disturbances caused by factors such as oxidative stress might jeopardize the viability of the lens cells, possibly resulting in opacification of the lens. Some studies have demonstrated that many different factors such as oxidative stress and UV irradiation can cause cataract formation, both in vivo and in vitro (Bloemendal, 1992).

\section{The cornea}

The cornea fulfills mainly a protective and refractive function, as it is located on the outermost surface of the eye. It is a highly transparent and strong tissue, separated from the surrounding conjunctiva by a transitional zone, the limbus. During eye development, the cornea is the last part of the eye to be formed. It consists of a stratified epithelium at the surface, stroma in the middle-populated by keratocytes and fibroblast-like cells - and an inner layer of endothelial cells. The layers are separated by Bowman's and Descemet's membranes (the former between the outer epithelium and stroma, the latter, between the stroma and inner epithelium or the so called endothelium of the cornea).

The formation of the sclera, cornea, and the uveal tunic has important ramifications for the nature of the fully formed eye. Since the mesoderm is the only embryonic tissue with angiogenic potential, i.e., the capacity to form blood vessels, its participation in the formation of the uveal tunic and sclera is indispensable. The cornea, although it fuses with the sclera, is derived solely from ectoderm (except in the endoderm), and it is, therefore, avascular in its final form. This lack of vascularization is of clinical significance because it isolates the cornea from the immune system, creating a "privileged site" suitable for transplantation.

The corneal epithelium is initially a continuation of the surface ectoderm, but in later development it arises from the superficial cells of the corneal stroma. Bowman's membrane is thickened and located on the long axis of the corneal surface. The corneal stroma develops from in situ mesenchymal tissue and not from the limbal mesenchymal tissue. The attenuation of the central cornea, early in the development, is due to the impingement of the lens against the developing cornea. Descemet's membrane is formed by the deep mesenchymal cells of the corneal stroma. The membrane is initially cellular and later becomes a homogeneous structure.
Fig 4. Lens cell differentiation. Diagram adapted from MavAvoy and Chamberlain (1989). The gradient of FGF stimulation may determine antero-posterior patterns of lens cell behavior. In the postnatal lens, cell proliferation is restricted to the epithelium and occurs predominantly in a band of cells above the equator (the germinative zone). The cellular behaviors are indicated (proliferation in orange, migration in red, and fiber differentiation in blue) in sequence as the concentration of FGF increases. The right-hand arrow indicates the proposed gradient of FGFstimulationgoverning this antero-posterior pattern of cell behavior in vivo. The left-hand arrow indicates that other factors, including inhibitory influences, may also contribute to the FGF gradient.
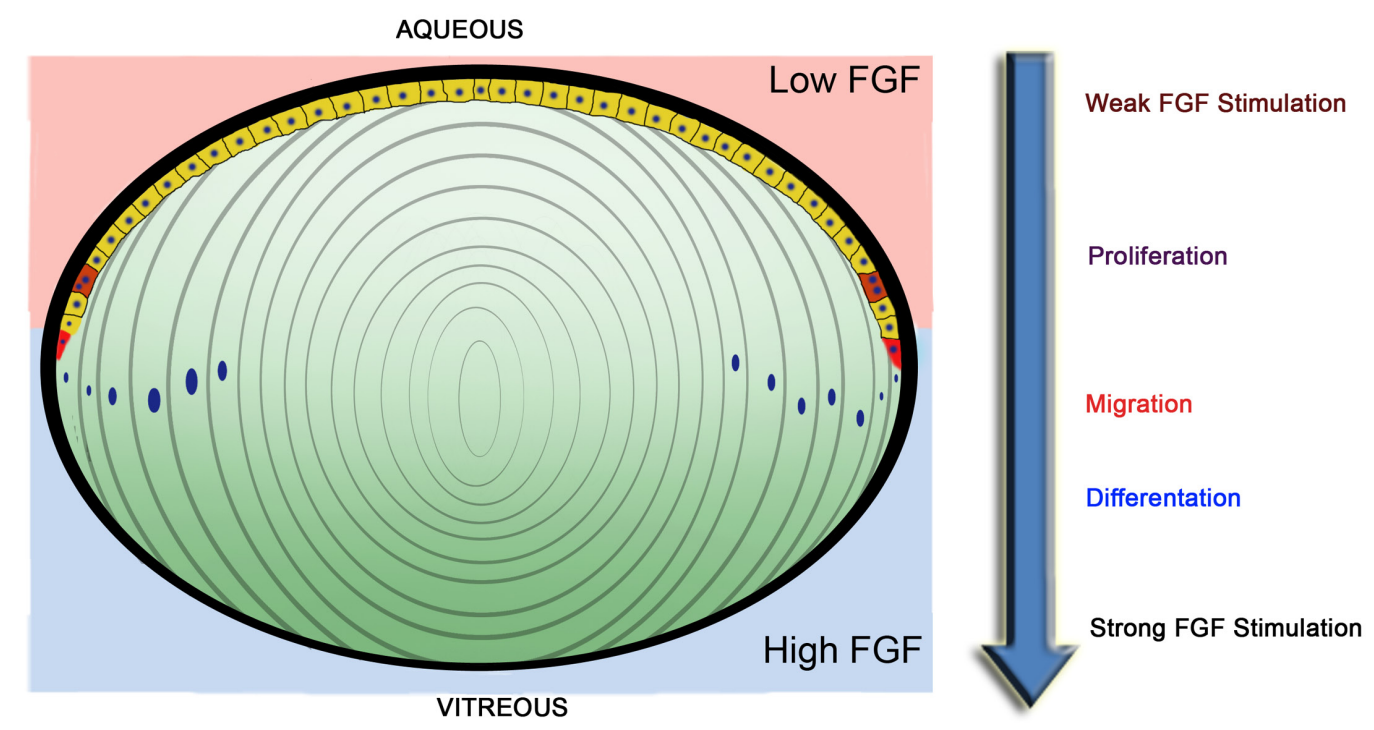
The endothelium is derived from the mesenchymal cells of the posterior stroma (Fig. 1). Tunica vasculosa lentis may play an important role during the early development of the cornea. It has been suggested that the anterior chamber is initially maintained by a transudate from this extensive capillary network. The aqueous humor develops with the regression of this vascular system. The hydrodynamic force of aqueous humor assists the corneal molding; the length and width of corneal stromal fibers increase corneal transparency and is maintained by extracellular matrix secreted by stromal keratocytes. Recently, Chan et al., have identified some genes implicated in the differentiation of human pluripotent embryonic stem cells (hES) into keratocytes in vitro, some of these genes are Ngfr, Snai1, Ntrk3, Sox9, and Msx1 (Chan AA et al., 2013). The results of their study could elucidate the keratocyte developmental pathway and help in the development of treatment for corneal stromal opacities.

Corneal epithelial stem/progenitor cells or limbal stem/progenitor cells (LSCs) are located at the limbus. These progenitor cells differentiate into trans-amplifying cells, which migrate centripetally towards the central cornea (Lavker RM et al., 2004). Mature corneal epithelial cells maintain the outer corneal surface. The LSC niche, similarly to the niches of other tissues, might have a key role in maintaining LSCs in an undifferentiated state.

The LSC niche contains several critical components that regulate LSC function, including the extracellular matrix (ECM), the limbal vasculature, and various supporting cells. These components provide the LSCs with specific signals for LSC homeostasis, using soluble factors and various cell-cell/matrix interactions. Several types of cells in the limbal region might function as LSC-niche cells, including limbal mesenchymal cells (LMCs), melanocytes, vascular endothelial cells, and smooth muscle cells. All these niche components might have a role in the growth and regulation of LSCs. During LSC deficiency, either due to congenital defect or after an injury, conjunctival cells might migrate onto the corneal surface in the absence of normal corneal epithelium. The preferred (and usually successful) treatment for this condition, limbal stem cell deficiency, is autologous transplantation of ex vivo expanded LSCs to the diseased eye (Rama RM et al., 2010).

In the development of the cornea and conjunctiva in humans, three stages can be distinguished: minimal proliferation (until 17th week), vigorous proliferation over the entire cornea including the limbus and the bulbar conjunctiva (until 28th week), and gradual decrease in proliferative activity (after 28th week) accompanied by the appearance of apoptotic cells.

Tissue repair in adult corneas elicits an elaborate cascade of responses, including recruitment of inflammatory cells, cytokinemediated apoptosis, activated proliferation, differentiation of repair myofibroblasts, and remodeling of ECM components (Sundarraj $\mathrm{N}$ et al., 1998). Tissue remodeling also takes place in the adult corneal stroma during the healing process, which may lead to scar formation and vision impairment (Hassel JR and Birk DE, 2010). Embryonic cornea regenerates rapidly with no detectable scar formation. Several mechanisms have been proposed for the scar-free regeneration of fetal wounds. The level of inflammation during the healing process is crucial in determining the magnitude of scarring. Wounded adult tissues undergo significant granulation and inflammation during repair, whereas the fetal wounds exhibit minimal inflammation (Larson BJ et al., 2010). In addition, fetal fibroblasts coordinate the synthesis and remodeling of ECM pro- teins, resulting in fiber orientations conducive to scar-free tissue regeneration.

The first cellular responses detected after a trauma to the adult cornea are the cytokine-induced apoptosis and proliferation of keratocytes triggered by the damage to the corneal epithelium and its basement membrane (Wilson SE, et al., 1996). Within 4 hours after injury, a large number of keratocytes at the wounded site undergo apoptosis, which is the earliest indicator of the wound-healing response. Even after an increase in wound size in the embryonic cornea, there is no increase in apoptosis in the epithelium and stroma during healing. Decrease in cellular density of keratocytes in the wounded corneas can be attributed to stretching of the wound as a result of elevated intraocular pressure and eye growth. With the exception of the regenerating epithelium, cell proliferation is lower in the wounded corneas than in stage-matched controls. Augmented cell proliferation in the corneal epithelium is probably due to re-epithelialization and induced by a process that does not affect the keratocytes. The absence of increased apoptosis and keratocyte proliferation suggests that the pro-inflammatory cytokines associated with these cellular responses in the adult corneal wounds are not active in the embryonic wound. Therefore, the absence of increased keratocyte turnover at the wound site may contribute to scar-free regeneration.

\section{Molecular pathways involved in cell death during eye development}

One of the processes involved in the early stages of development is the activation of pro-apoptotic pathways. These processes represent the most common mechanisms regulating the size of cell populations in embryos and adults.

Apoptosis (derived from a Greek word meaning "falling off", as leaves fall from a tree in the autumn) is the main morphological feature of the process of PCD or "cell suicide" (Oppenheim, 1991).

In the developing vertebrate nervous system, half or more of the nerve cells normally die soon after they are born. Moreover, deregulated cell death is increasingly recognized as a frequent factor in different diseases. This process is the final common pathway triggered by a variety of primary defects, including various neurodegenerative diseases. Apoptosis is a difficult process to measure because the dead cells are rapidly degraded by their neighbors. There are still many process to be studied in relation to the molecular mechanisms targeting only certain cells for apoptosis and those integrating it with other biological processes

A variety of stimuli can lead to apoptotic cell death. These proapoptotic stimuli include both extracellular and intracellular signals. The former include TNF, FasL, or the absence of trophic factors, while DNA damage and ER stress are examples of intracellular proapoptotic signals. These specific signals activate the intracellular pathways and cause biochemical and morphological changes in the cell. Caspases, the enzymes cleaving their target proteins at specific aspartic acid residues, are typically activated during the early stages of apoptosis.

It is clear that apoptosis events that take place in the retina are crucial, while apoptosis in other eye tissues is not a key event but a refinement. Thus, for the final development of the cornea, the control of the keratocyte proliferation is more important than the cell death events. However, in the retina, the PCD has several possible key functions. These functions are: (1) the elimination of 
neurons that fail to make functional synaptic contacts with their targets, (2) elimination of cells or separation of cell layers during epithelial sheet fusion and shape change, and (3) elimination of transient populations that have an important function only during a particular phase of development.

The cell death program seen in normal eye development is also triggered after injury and in disease states. The molecular mechanisms underlying the apoptotic cell death have been conserved throughout the evolution.

\section{Acknowledgements}

Grupos Consolidados Gobierno Vasco GOBE (IT437-10) and Red Patología Ocular (RETICS-RD07/0062) are gratefully acknowledged.

\section{References}

AGATHOCLEOUS M and HARRIS WA (2009). From progenitors to differentiated cells in the vertebrate retina. Annu Rev Cell Dev Biol 25: 45-69.

AHMAD I, DAS AV, JAMES J, BHATTACHARYA S and ZHAO X (2004). Neural stem cells in the mammalian eye: types and regulation. Semin Cell Dev Biol 15: 53-62.

ANG SJ, STUMP RJ, LOVICU FJ and MCAVOY JW (2004). Spatial and temporal expression of Wnt and Dickkopf genes during murine lens development. Gene Expr Patterns 4: 289-295.

BÄHR, M. (2000). Live or let die-retinal ganglion cell death and survival during development and in the lesioned adult CNS. Trends Neurosci 23: 483-490.

BEEBE D C, FEAGANS D E and JEBENS H A (1980). Lentropin: a factor in vitreous humor which promotes lens fiber cell differentiation. Proc. Natl. Acad. Sci. USA 77: 490-493.

BLOEMENDAL, H. (1992). Disorganization of membranes and abnormal intermediate filament assembly lead to cataract. Proctor Award Lecture. Invest. Ophthalmol. Visual Sci 32: 445-455.

CAVODEASSI F, CARREIRA-BARBOSA F, YOUNG RM, CONCHA ML, ALLENDE ML, HOUART C, TADA M and WILSON SW (2005). Early stages of zebrafish eye formation require the coordinated activity ofWnt11, Fz5, and the Wnt/beta-catenin pathway. Neuron 47: 43-56.

CHAN AA, HERTSENBERG AJ, FUNDERBURGH ML, MANN MM, DU Y, DAVOLI KA, MICH-BASSO JD, YANG L and FUNDERBURGH JL (2013). Differentiation of human embryonic stem cells into cells with corneal keratocyte phenotype. PLoS One 8:e56831.

CLARKE, P.G.H. (1985). Neuronal death in the development of the vertebrate nervous system. Trends Neurosci 8: 345-349.

DAHL E, KOSEKI H and BALLING R (1997). Pax genes and organogénesis. Bioessays 19: 755-765.

DE MELO J., QIU X, DU G, CRISTANTE L and EISENSTAT DD (2003). DIx1, DIx2, $P a x 6, B r n 3 b$, and $C h x 10$ homeobox gene expression defines the retinal ganglion and inner nuclear layers of the developing and adult mouse retina. J. Comp. Neurol. 461: 187-204.

DEGTEREV Aand YUANJ (2008) Expansion and evolution of cell death programmes. development in chick. Dev Neurosci. 2004; 26:456-65.

DIASDASILVAMR, TIFFIN N, MIMAT, MIKAWAT and HYER J (2007). FGF-mediated induction of ciliary body tissue in the chick eye. Dev Biol 304: 272-285.

DÜNKER N and KRIEGLSTEIN K (2003) Reduced programmed cell death in the retina and defects in lens and cornea of TgfB2 TgfB3 double-deficient mice. Cell Tissue Res 313: 1-10.

FABER S.C, DIMANLIG P, MAKARENKOVA H.P, SHIRKE S, KO K and LANG R.A (2001). Fgf receptor signaling plays a role in lens induction. Development 128: 4425-4438.

FISHER LJ (1979). Development of synaptic arrays in the inner plexiform layer of neonatal mouse retina. J Comp Neurol 187: 359-372.

GALLI-RESTA, L. and ENSINI, M. (1996). An intrinsic limit between genesis and death of individual neurons in the developing retinal ganglion cell layer. J. Neurosci 16: 2318-2324.

GARCIA CM, HUANG J, MADAKASHIRA BP, LIU Y, RAJAGOPAL R, DATTILO L, ROBINSON ML and BEEBE DC (2011). The function of FGF signaling in the lens placode. Dev Biol 351: 176-85.

GOUDREAU G, ANDREJEWSKI N and GRUSS P (2004). Transcription factors in early lens development. F.J. Lovicu, M.L. Robinson (Eds.). Development of the ocular lens, Cambridge Univ. Press, New York.

HASSEL JR and BIRK DE (2010). The molecular basis of corneal transparency. Exp Eye Res 91: 110.

ISSENMANN, S., KRETZ, A. and CELLERINO, A. (2003). Molecular determinants of retinal ganglion cell development, survival, and regeneration. Prog. Ret. Eye Res 22: 483-543.

KERR CL, ZAVERI MA, ROBINSON ML, WILLIAMS T and WEST-MAYS JA (2014). -AP-2 $\alpha$ is required after lens vesicle formation to maintain lens integrity. Dev Dyn 243: 1298-309.

LANG R and MCAVOY J.W (2004). Growth factors in lens development. F.J. Lovicu, M.L. Robinson (Eds.), Development of the ocular lens, Cambridge Univ. Press, New York.

LARSONBJ, LONGAKERMT and LORENZ HP (2010). Scarless fetal wound healing: a basic science review. Plast Reconstr Surg 126: 1172-1180.

LAVKER RM, TSENG SCG and SUN T-T (2004). Corneal epithelial stem cells at the limbus: looking at some old problems from a new angle. Exp Eye Res 78: 433-446.

LINDEN R (1994). The survival of developing neurons-a review of afferent control. Neurosci. 85: 671-682.

LIU H, THURIG S, MOHAMED O, DUFORT D and WALLACE VA (2006). Mapping canonical Wnt signaling in the developing and adult retina. Invest Ophthalmol Vis Sci 47: 5088-5097

MEYER-FRANKE A, WILKINSON G A, KRUTTGEN A, HU M, MUNRO E, HANSON JR, REICHARDT MG and BARRES, B A (1998). Depolarization and camp elevation rapidly. Nat Rev Mol Cell Biol 9: 378-390.

OHSAWA R and KAGEYAMA R (2008). Regulation of retinal cell fate specification by multiple transcription factors. Brain Research 1192: 90-98.

OPPENHEIM, R W (1991). Cell death during development of the nervous system. Annu. Rev Neurosci 14: 453-501.

PATTERSON P H (1992). Neuron-target interactions. In Z Hall ed. An introduction to Molecular Neurobiology. Sinauer Associates, Sunderland, MA, pp 428-459.

PERRY V H, HENDERSON Z and LINDEN R (1983). Postnatal changes in retinal ganglion and optic axon populations in the pigmented rat. J. Comp. Neurol 219: 356-368.

QUIRING R, WALLDORF U, KLOTER U and GEHRING WJ (1994). Homology of the eyeless gene of Drosophila to the Small eye gene in mice and Aniridia in humans. Science. 265: 785-789.

RAFF M C, BARRES B A, BURNE J F, COLES H S, ISHIZAKI Y and JACOBSON $M D$ (1993). Programmed cell death and the control of cell survival: lesson from the nervous system. Science 262: 695-700.

RAGER G and RAGERU (1978). Systems-matching by degeneration. I. A quantitative electron microscopic study of the generation and degeneration of retinal ganglion cells in the chicken. Exp. Brain Res 33: 65-78.

RAMA P, MATUSKA S, PAGANONI G, SPINELLI A, DE LUCA M and PELLEGRINI $G$ (2010). Limbal stem-cell therapy and long-term corneal regeneration. N Engl J Med 363: 147-155.

REH T A and FISCHER A J (2006). Retinal stem cells. Methods in Enzymology 419: 52-73.

SUNDARRAJ N, FITE D, BELAK R, SUNDARRAJ S, RADA J, OKAMOTO $S$ and HASSELL J (1998). Proteoglycan distribution during healing of corneal stromal wounds in chick. Exp Eye Res 67: 433-442.

TALEBIZADEH N, YU Z, KRONSCHLÄGER M, HALBÖÖK F and SÖDERBERG $P$ (2014). Specific spatial distribution of caspase-3 in normal lenses. Acta Ophthalmol 14: 1111.

THOMAIDOU D, MIONE M C, CAVANAGH J F R and PARNAVELAS J G (1997). Apoptosis and its relation to the cell cycle in the developing cerebral cortex. $J$ Neurosci 17: 1075-1085.

TORNBERRY, N.A. and LAZEBNIK, Y. (1998). Caspases: enemies within. Science 281: 1312-1316.

TROUSSE F, ESTEVE P and BOVOLENTA P (2001). BMP4 mediates apoptotic cell death in developing chick eye. J. Neruosci 21: 1292-1301.

VECINO E, HERNADEZ M and GARCÍA M (2004). Cell death in the developing vertebrate retina. Int. J. Dev. Biol 48: 965-974. 
WILSON S E, HE Y G, WENG J, LI Q, MCDOWALL A W, VITAL M, CHWANG E L (1996). Epithelial injury induces keratocyte apoptosis: hypothesized role for the interleukin-1 system in the modulation of corneal tissue organization and wound healing. Exp Eye Res 62: 325-337.

YAMAMOTO A, NAGANO T, TAKEHARA S, HIBI M and AIZAWA S (2005). Shisa promotes head formation through the inhibition of receptor protein maturation for the caudalizing factors, Wnt and FGF. Cell.120: 223-235.

YU H, SMALLWOOD P M, WANG Y, VIDAL T R, REED R. and NATHANS, J. (2010)
Frizzled 1 and frizzled 2 genes function in palate, ventricular septum and neura tube closure: general implications for tissue fusion processes. Development 137: 3707-3717.

ZHANG L, YAN Q, LIU J P, ZOU L J, LIU J, SUN S, DENG M, GONG L, JI W K and LI D W (2010). Apoptosis: its functions and control in the ocular lens. Curr Mol Med 10: 864-875.

ZUBER M E, GESTRI G, VICZIAN A S, BARSACCHI G and HARRIS W A (2003). Specification of the vertebrate eye by a network of eye field transcription factors. Development 130: 5155-5167. 


\section{Further Related Reading, published previously in the Int. J. Dev. Biol.}

Apoptosis in Drosophila: compensatory proliferation and undead cells Francisco A. Martín, Ainhoa Peréz-Garijo and Ginés Morata Int. J. Dev. Biol. (2009) 53: 1341-1347

NMDA-receptor blockade enhances cell apoptosis in the developing retina of the postnatal rat María Hernández, Inmaculada Guerrikagoitia, Luis Martínez-Millan and Elena Vecino Int. J. Dev. Biol. (2007) 51: 117-122

Retinal ganglion cells: dying to survive GUERIN MB, MCKERMAN, DP, O'BRIEN CJ, COTTER TG Int J Dev Biol. (2006) 50: 665-74.

Cell death in the developing vertebrate retina. VECINO E, HERNADEZ M and GARCÍA M. Int. J. Dev. Biol (2004) 48: 965-974.

New views on retinal axon development: a navigation guide.

MANN F, HARRIS WA, HOLT CE.

Int J Dev Biol. (2004) 48: 957-64.
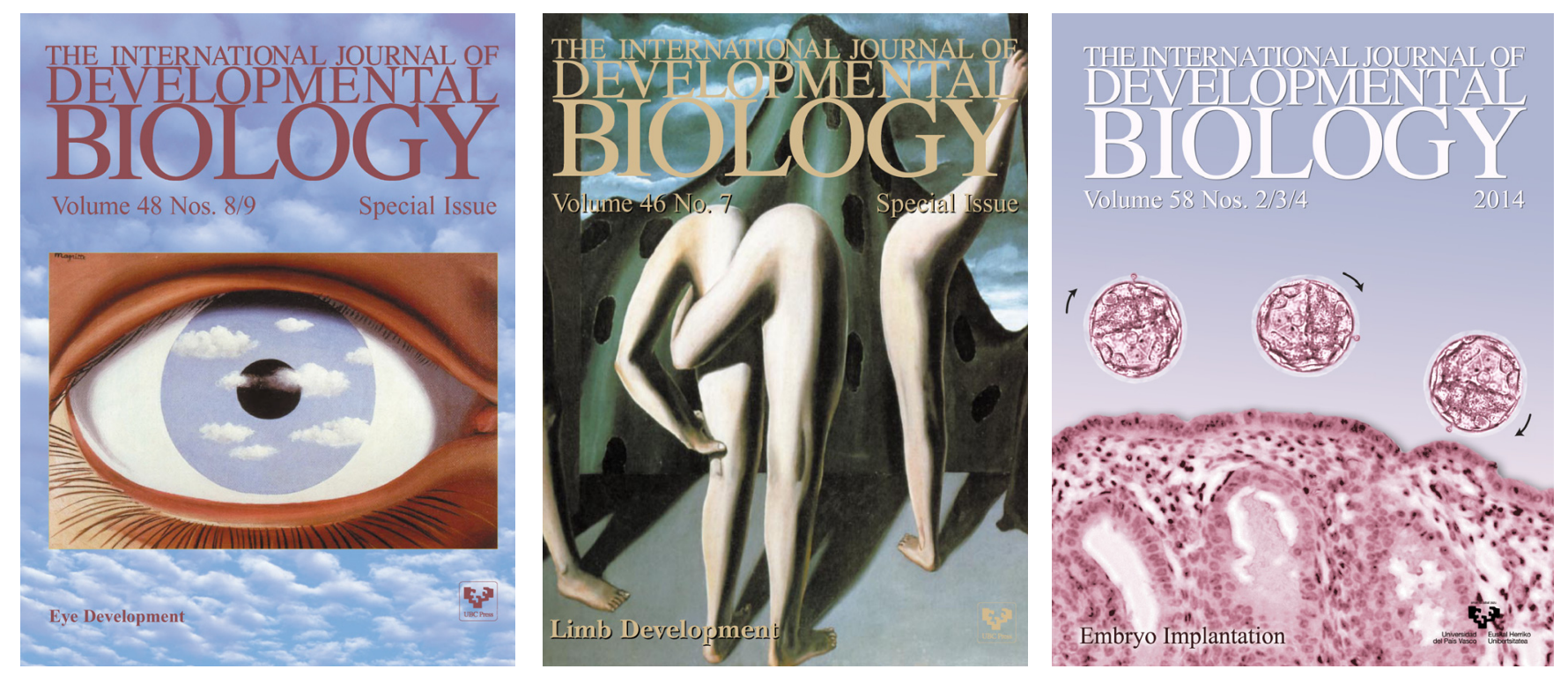